\title{
Giant Magnetic Susceptibility of Gold Nanorods Detected by Magnetic Alignment
}

\author{
P. G. van Rhee, ${ }^{1}$ P. Zijlstra, ${ }^{2}$ T. G. A. Verhagen, ${ }^{3}$ J. Aarts,${ }^{3}$ M. I. Katsnelson, ${ }^{4}$ J. C. Maan, ${ }^{1}$ \\ M. Orrit, ${ }^{2}$ and P.C. M. Christianen ${ }^{1, *}$ \\ ${ }^{1}$ High Field Magnet Laboratory, Institute of Molecules and Materials, Radboud University Nijmegen, \\ Toernooiveld 7, 6525 ED Nijmegen, Netherlands \\ ${ }^{2}$ Institute of Physics, Leiden University, P.O. Box 9504, 2300 RA Leiden, Netherlands \\ ${ }^{3}$ Kamerlingh Onnes Laboratory, Leiden University, P.O. Box 9504, 2300 RA Leiden, Netherlands \\ ${ }^{4}$ Institute of Molecules and Materials, Radboud University Nijmegen, Heyendaalseweg 135, 6525 AJ Nijmegen, Netherlands
} (Received 19 July 2013; published 17 September 2013)

\begin{abstract}
We have determined the magnetic properties of single-crystalline Au nanorods in solution using an optically detected magnetic alignment technique. The rods exhibit a large anisotropy in the magnetic volume susceptibility $\left(\Delta_{V}\right) . \Delta \chi_{V}$ increases with decreasing rod size and increasing aspect ratio and corresponds to an average volume susceptibility $\left(\chi_{V}\right)$, which is drastically enhanced relative to bulk Au. This high value of $\chi_{V}$ is confirmed by SQUID magnetometry and is temperature independent (between 5 and $300 \mathrm{~K}$ ). Given this peculiar size, shape, and temperature dependence, we speculate that the enhanced $\chi_{V}$ is the result of orbital magnetism due to mesoscopic electron trajectories within the nanorods.
\end{abstract}

DOI: 10.1103/PhysRevLett.111.127202

PACS numbers: 75.75.-c, 73.22.-f, 75.20.En, 78.67.Qa

Bulk $\mathrm{Au}$ is a diamagnetic material, i.e., one with a negative volume magnetic susceptibility $\chi_{\mathrm{Au}}$. Recently, it was reported that Au nanoparticles (NPs), with functionalized surfaces, show a broad range of magnetic behavior, ranging from (enhanced) diamagnetic [1,2] to (super)paramagnetic [3-5] and even ferromagnetic up to room temperature [6,7]. The NP size and the type of capping molecules, strongly binding to or weakly interacting with $\mathrm{Au}$, appear to influence the magnetic response. Several explanations were suggested, such as competing magnetic contributions of the NP core and surface [3], the formation of a magnetic moment due to the exchange of charges at the Au-ligand interface $[5,6,8]$, the creation of large orbital moments due to electron motion within surface clusters [9], and the occurrence of persistent currents in the $\mathrm{Au}$ core [2]. However, so far, the origin of this unexpected magnetism and why it differs strongly between different types of NPs is not yet understood [2,10,11].

We employ a novel magnetic alignment technique to measure the magnetic properties of rod-shaped Au NPs in solution. We focus on relatively large NPs (all dimensions $>7 \mathrm{~nm}$ ) that are single crystalline. The degree of alignment is measured optically, through the magnetic field-induced linear dichroism and birefringence, across the $\mathrm{Au}$ surface plasmon resonance (SPR) that arises due to collective oscillation modes of the conduction electrons [12,13]. We find an enhanced (dia)magnetic behavior, which does not depend on temperature (in the range 5-300 K). We speculate that this enhanced magnetism is an orbital effect, resulting from mesoscopic electron trajectories within the NPs [2,14].

The optically detected magnetic alignment technique relies on the anisotropy of both the optical and magnetic properties of the Au nanorods. Because of their shape, the rods exhibit an anisotropic optical response, determined by their longitudinal $\left(\alpha_{\|}\right)$and transverse $\left(\alpha_{\perp}\right)$ polarizabilities [15]. Polarized light, therefore, provides a sensitive tool to determine the alignment of rods [16-19]. In this Letter, rod alignment is induced by a magnetic field $(\vec{B})$ because of the difference in the magnetic susceptibility parallel $\left(\chi_{\|}\right)$and perpendicular $\left(\chi_{\perp}\right)$ to the long rod axis. This leads to an orientational magnetic energy that depends on the angle ( $\theta)$ of the rod axis relative to the field direction: $\Delta E_{m}=$ $-\left(\Delta \chi_{V} V B^{2} \cos ^{2} \theta / 2 \mu_{0}\right)$, with $\Delta \chi_{V}=\chi_{\|}-\chi_{\perp}, V$ the NP volume, and $\mu_{0}$ the magnetic constant. When $\Delta E_{m}$ is of the order of the thermal energy $k_{B} T$, this will result in alignment, which follows a Boltzmann distribution function $\sim \exp \left[-\Delta E_{m}(\theta) / k_{B} T\right]$ and which can be characterized by an order parameter $S$ quantifying the degree of alignment. $S$ is positive (negative) for alignment along (perpendicular to) the magnetic field, where $|S| \propto B^{2}$ at low fields (because $\Delta E_{m} \propto B^{2}$ ) until it saturates at high fields in the case of complete alignment [15]. By measuring the magnetic field-induced linear dichroism (LD) and linear birefringence (LB), the anisotropy of the magnetic susceptibility $\Delta \chi_{V}$ is measured (see below). Our optical method permits us to selectively probe the magnetism $\left(\Delta_{\chi_{V}}\right)$ of isolated $\mathrm{Au}$ NPs in solution at concentrations that are orders of magnitude smaller than those required for measurements using a superconducting quantum interference device (SQUID) magnetometer [1,6,7].

A set of 10 colloidal Au nanorod samples was prepared by silver-assisted seed-mediated growth [20,21], a method resulting in high quality, single-crystalline [15], spherically capped cylindrical NPs [22]. By varying both the silver-ion and seed concentrations, the aspect ratio $A R$ and volume $V$ of the nanorods were varied. The rods were coated with either cetyl trimethylammonium bromide (CTAB) or thiolated polyethylene glycol (mPEG-SH, 
molecular weight $5 \mathrm{kDa}$ ) to make them water soluble and to prevent clustering. Each sample was extensively characterized by scanning electron microscopy (SEM) [Fig. 1(a)] to determine the average length $L$, width $W$, and aspect ratio $A R=L / W$ [Fig. 1(b), Table I]. The NPs used are relatively large (all dimensions in the range of $7-73 \mathrm{~nm}$ ) but still smaller than or in the range of the mean free path of bulk $\mathrm{Au}$ at room temperature $(\sim 60 \mathrm{~nm}$ $[23,24])$. The extinction spectra of aqueous rod solutions exhibit well-defined SPR peaks, consisting of a transversal peak (collective electron motion along $W$ ) around $520 \mathrm{~nm}$ and a longitudinal peak (collective electron motion along $L)$ that shifts towards longer wavelengths with increasing $A R$ [Fig. 1(c)] [12,13]. The selectivity of our optically detected magnetic alignment technique is caused by this wavelength and orientation dependence of the SPR.

We measure $S(B)$, and thereby $\Delta_{\chi_{V}}$, by LD and LB on aqueous $\mathrm{Au}$ rod suspensions in an optical cuvette positioned in a $33 \mathrm{~T}$ Florida-Bitter magnet. The measured LD signal is given by the difference in the extinction of light polarized parallel and perpendicular to the field direction, given by [15]

$$
\Delta A(B, \lambda)=A_{\|}-A_{\perp} \propto S(B) \operatorname{Im}\left[\alpha_{\|}(\lambda)-\alpha_{\perp}(\lambda)\right] .
$$

Similarly, LB measures the difference in the refractive index of light polarized parallel and perpendicular to $\vec{B}$, given by [15]

$$
\Delta n(B, \lambda)=n_{\|}-n_{\perp} \propto S(B) \operatorname{Re}\left[\alpha_{\|}(\lambda)-\alpha_{\perp}(\lambda)\right] .
$$

We use sample $H$ (Table I) to illustrate the typical results. Figure 2(a) shows the $\Delta A(B)$ signal for several wavelengths. All curves display a $B^{2}$ dependence up to the highest field used. The up and down sweeps lead to identical curves, without hysteresis, independent of sweep rate and light intensity. The size of the LD signal at $30 \mathrm{~T}$ [symbols in Fig. 2(b)] roughly follows $A(0, \lambda)$, shown by the dashed red line. For wavelengths within the longitudinal SPR, the LD signal is positive $\left(A_{\|}>A_{\perp}\right)$, whereas at the transverse SPR, the signal is negative $\left(A_{\|}<A_{\perp}\right)$. This clearly demonstrates that the rods align with their long axis along the field.

The LB amplitude at $30 \mathrm{~T}$ [symbols in Fig. 2(d)] changes sign when crossing the SPR maximum, indicating the derivativelike spectrum of the refractive index. The LB signal increases linearly with rod concentration [15]. It proves that the magnetic field induces alignment of isolated rods, without any trace of rod aggregation, up to the highest rod concentrations used. To determine the pure degree of magnetic alignment of the rods, we take into account the wavelength and concentration dependence of the signal by defining the order parameter as $S(B)=$ $\Delta A(B, \lambda) / \Delta A_{\max }(\lambda) . \Delta A_{\max }(\lambda)$ is the maximum $\Delta A$ at a given wavelength, which is reached in the case of full alignment. For the longitudinal case, it is given by $\Delta A_{\max }(\lambda) \approx 3 A(0, \lambda)[25]$. The resulting $S(B)$ thus reflects
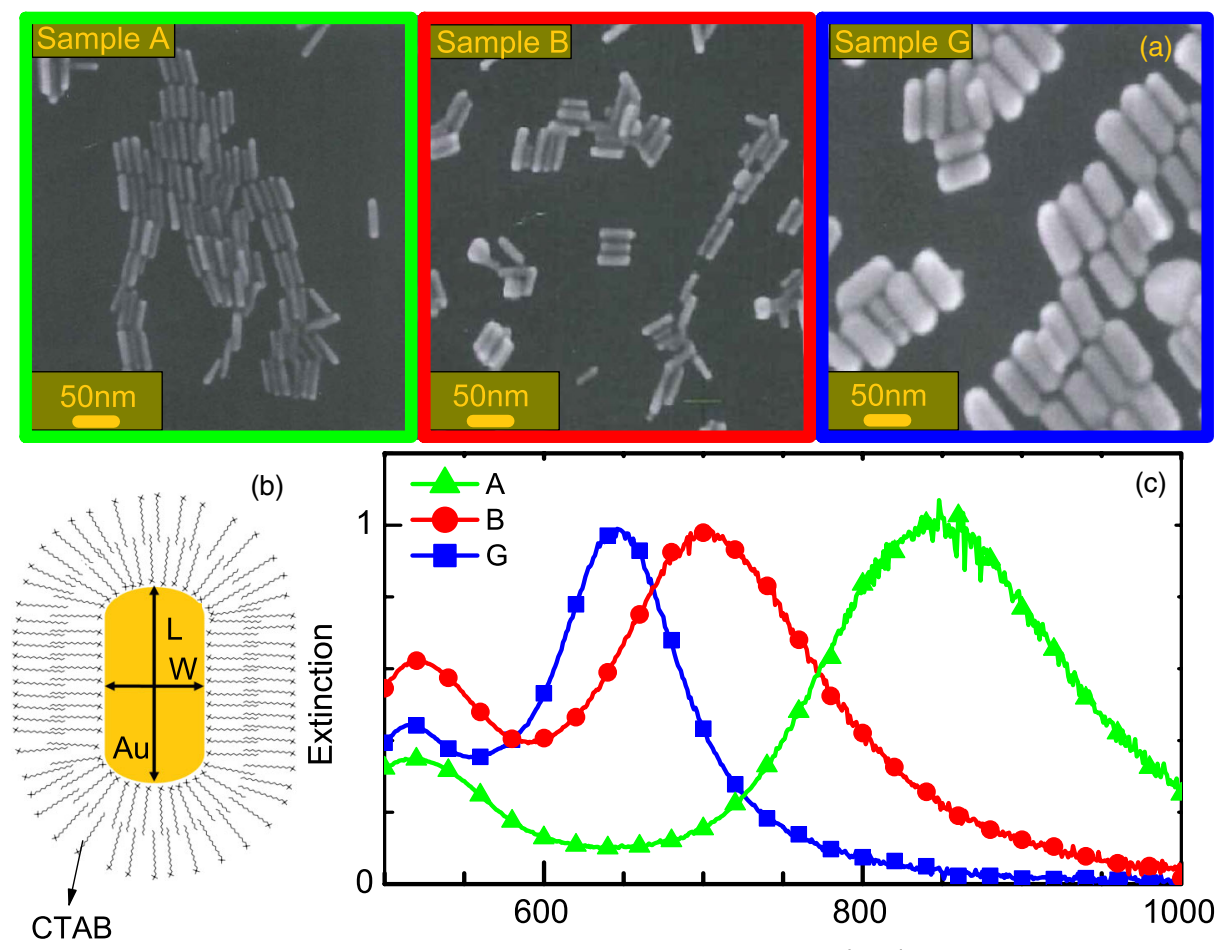

(b)
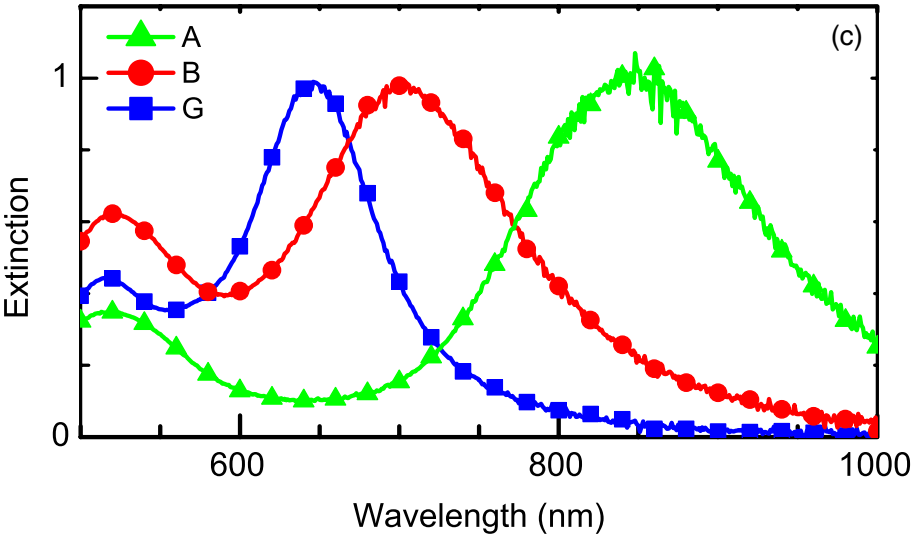

FIG. 1 (color online). (a) SEM images of Au nanorod samples $A, B$, and $G$. The images were obtained after dropcasting a $2 \mu 1$ droplet of a $0.1 \mathrm{nM}$ nanorod-water solution on a conducting Si substrate. (b) Schematic drawing of a cylindrical CTAB-capped Au nanorod with length $L$ and width $W$. (c) Normalized extinction spectra of samples $A, B$, and $G$. 
TABLE I. Sample properties: length $L$, width $W$, aspect ratio $A R=L / W$, volume $V=4 \pi W^{3} / 3+(L-W) \pi W^{2}$, rod concentration $c_{\text {rod }}$, measured order parameter $S(32 \mathrm{~T})$, and the corresponding calculated susceptibility difference $\Delta \chi_{V}$ [15]. All samples have a CTAB coating, except sample $J_{P}$, which has a mPEG-SH coating.

\begin{tabular}{|c|c|c|c|c|c|c|c|}
\hline \# & $L \mathrm{~nm}$ & $W \mathrm{~nm}$ & $A R$ & $V 10^{3} \mathrm{~nm}^{3}$ & $c_{\text {rod }} \mathrm{nM}$ & $S(32 \mathrm{~T})$ & $\Delta \chi_{V} 10^{-8}$ \\
\hline$A$ & $35 \pm 3$ & $7 \pm 1$ & 5.0 & $1.2 \pm 0.5$ & 1.60 & 0.015 & 92 \\
\hline$B$ & $33 \pm 6$ & $9 \pm 2$ & 3.7 & $2.0 \pm 0.8$ & 0.96 & 0.017 & 68 \\
\hline$C$ & $31 \pm 5$ & $12 \pm 2$ & 2.6 & $3.1 \pm 1.5$ & 1.17 & 0.011 & 28 \\
\hline$D$ & $44 \pm 7$ & $15 \pm 3$ & 3.0 & $6.5 \pm 3.7$ & 0.44 & 0.013 & 16 \\
\hline$E$ & $52 \pm 5$ & $18 \pm 3$ & 2.8 & $12 \pm 5$ & 0.26 & 0.048 & 29 \\
\hline$F$ & $60 \pm 6$ & $22 \pm 4$ & 2.7 & $20 \pm 9$ & 0.17 & 0.036 & 13 \\
\hline$G$ & $60 \pm 6$ & $25 \pm 4$ & 2.5 & $26 \pm 11$ & 0.15 & 0.034 & 10 \\
\hline$H$ & $73 \pm 8$ & $31 \pm 5$ & 2.4 & $48 \pm 21$ & 0.08 & 0.081 & 12 \\
\hline$I$ & $40 \pm 8$ & $15 \pm 5$ & 2.7 & $6 \pm 3$ & 0.65 & 0.032 & 39 \\
\hline$J_{P}$ & $40 \pm 8$ & $15 \pm 5$ & 2.7 & $6 \pm 3$ & 0.65 & 0.026 & 32 \\
\hline
\end{tabular}

the magnetic alignment of the rods and does not depend on the rod concentration or the wavelength used.

Figure 2(c) shows the resulting $S(B)$ for several samples. In all cases, $S>0$, indicating rods aligning parallel to the field. $S$ increases quadratically with $B$ with a maximum (at $32 \mathrm{~T}$ ) that is sample dependent [see results in Table I and Fig. 3(a)] but which never reaches complete alignment $(S=1)$. Sample $H$ reaches an order parameter of $S=$ 0.071 at $30 \mathrm{~T}$. Inserting this value in Eqs. (1) and (2) leads to the full description of the LD and LB responses at $30 \mathrm{~T}$. The LD and LB curves are interrelated by a KramersKronig transformation without any adjustable parameter [solid blue lines in Figs. 2(b) and 2(d)], which illustrates the internal consistency of our optical method [15]. We conclude that the magnetic moment linearly increases with
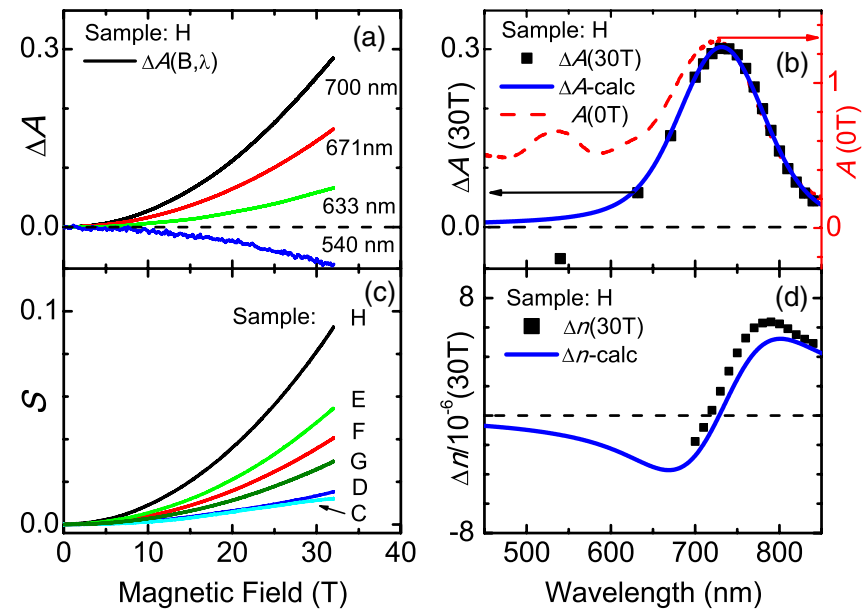

FIG. 2 (color online). (a) $\Delta A(B)$ of sample $H$ for different wavelengths. (b) LD measurement: Squares give $\Delta A(\lambda)$ at $30 \mathrm{~T}$, and it roughly follows $A(0, \lambda)$, the extinction spectrum at $0 \mathrm{~T}$, indicated by the dashed red line. The solid blue line shows the calculated longitudinal absorption $A_{\|}$(c) $S(B)$ for six different samples (Table I). (d) LB measurement: Black squares give $\Delta n(\lambda)$ at $30 \mathrm{~T}$, and the solid blue line shows the calculated longitudinal birefringence $n_{\|}$.
$B$ up to $32 \mathrm{~T}$, without hysteresis, indicative of a dia- or paramagnetic moment.

The symbols in Fig. 3(a) summarize the experimental results, revealing a maximum order parameter $[S(32 \mathrm{~T})]$ that increases with the rod volume. Since $S(32 \mathrm{~T}) \propto \Delta \chi_{V} V$ [right axis of Fig. 3(a)], we can use these results to obtain $\Delta \chi_{V}$ as a function of $V$ [symbols in Fig. 3(b)] and $A R$ [symbols in Fig. 3(c)]. We find $\Delta \chi_{V}$ values of the order of $10^{-6}-10^{-7}$ and $\Delta \chi_{V}$ increases with decreasing $V$ and increasing $A R$.

In a first attempt to describe these results, we assume that the magnetic susceptibility $\left(\chi_{V}\right)$ of the rods is uniform and that the magnetic alignment is merely caused by shape demagnetization effects. In the case of $\left|\chi_{V}\right| \ll 1, \Delta \chi_{V}$ is given by [15]

$$
\Delta \chi_{V}=\left(D_{\perp}-D_{\|}\right)\left(\chi_{V}-\chi_{m}\right)^{2},
$$

with $D_{\perp}$ and $D_{\|}$, the demagnetization factors perpendicular and parallel to the rod axis, respectively, and $\chi_{m}$ the susceptibility of the medium $\left(\chi_{m}=-8.8 \times 10^{-6}\right.$ for water [23]). Taking the demagnetization factors for ellipsoids [26] and inserting the bulk Au susceptibility $\left(\chi_{V}=\right.$ $\chi_{\mathrm{Au}}=-3.4 \times 10^{-5}[23]$ ), we find a $\Delta \chi_{V}$ value of about $10^{-10}$, which is 3 orders of magnitude too small compared to the data [dash-dotted green lines in Figs. 3(b) and 3(c)]. This result strongly suggests that the magnetic susceptibility of Au NPs is drastically enhanced over the bulk value.

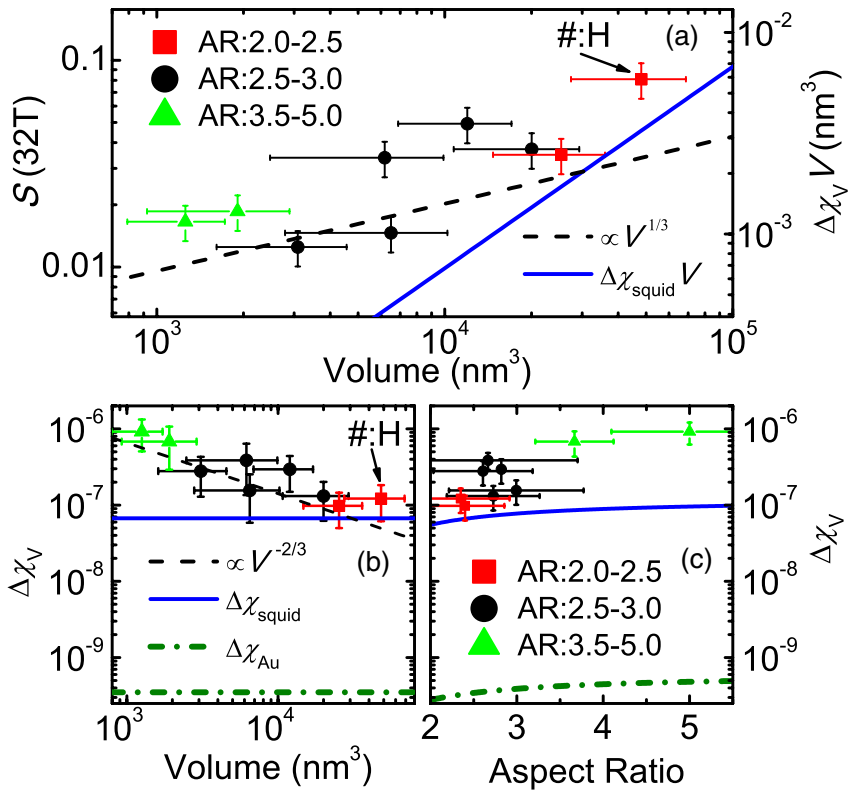

FIG. 3 (color online). (a) $S(32 \mathrm{~T})$ (and $\left.\Delta \chi_{V} V\right)$ as functions of $V$ for the nanorod samples. The dashed black line indicates $S \propto V^{1 / 3}$. (b) $\Delta \chi_{V}$ as a function of $V$. The dashed black line indicates $\Delta \chi_{V} \propto V^{-2 / 3}$ (c) $\Delta \chi_{V}$ as a function of $A R$. In all panels: Three different regimes of the $A R$ are labeled by the squares, circles, and triangles. The solid blue and dash-dotted green lines are calculated with Eq. (3), using, respectively, the measured SQUID susceptibility $\chi_{\text {SQUID }}$ of sample $H(: H)$ and the susceptibility $\chi_{\mathrm{Au}}$ of bulk Au. 
To test this hypothesis, we have measured $\chi_{V}$ of a high concentration $(185 \mathrm{nM})$ nanorods of sample $H$ (largest $V$ and $S$ ), dispersed into a polyvinyl alcohol matrix, by a SQUID magnetometer experiment. Figure 4 shows the background-corrected [15] magnetic moment per rod $m_{\text {rod }}$ as a function of magnetic field and temperature. $m_{\mathrm{rod}}(B)$ was found to be independent of temperature (between 5 and $300 \mathrm{~K}$ ) and linear with $B$, with a negative slope, showing no trace of saturation, demonstrating that the rods are diamagnetic with a magnetic susceptibility of $\chi_{V}=(-4.9 \pm 1.2) \times 10^{-4}$, i.e., $14( \pm 4) \times \chi_{\mathrm{Au}}$.

The outcome of the SQUID experiment demonstrates that the enhanced magnetism is not due to any paramagnetic impurities in the sample. Most importantly, it proves that the overall (bulk) magnetic susceptibility of sample $H$ is enhanced over $\chi_{\mathrm{Au}}$ by more than 1 order of magnitude. Inserting this enhanced $\chi_{V}$ value in Eq. (3) indeed leads to a $\Delta \chi_{V}=\Delta \chi_{\text {SQUID }}$ value for sample $H$ that is consistent with the outcome of the magnetic alignment experiment (solid blue curves in Fig. 3). The enhanced magnetism of sample $H$ is, therefore, a bulk effect and not the result of $\mathrm{Au}$ surface states $[27,28]$ or other geometrical effects $[14,29,30]$. It should be noted, however, that Eq. (3) underestimates the strong $V$ and $A R$ dependencies of the $\Delta \chi_{V}$ data [solid blue curves in Figs. 3(b) and 3(c)]. Finally, the SQUID experiment rules out that the alignment is due to the intrinsic anisotropy of the magnetic susceptibility of the CTAB capping molecules [31] because in that case $\Delta \chi_{V} \propto \chi_{V}[15,25,32,33]$. We have verified that solutions with varying CTAB concentrations (from 0.3 to $11.6 \mathrm{mM}$ ) but the same Au cores lead to identical LB signals [15]. Even the highest CTAB concentration is too low to form a liquid crystalline phase that could be magnetically aligned [17,31]. Furthermore, samples $I$ and $J_{P}$, which are identical and have only different capping molecules, show a very similar LD signal (not shown) [15]. The relative insensitivity of the signal to the capping molecules and the

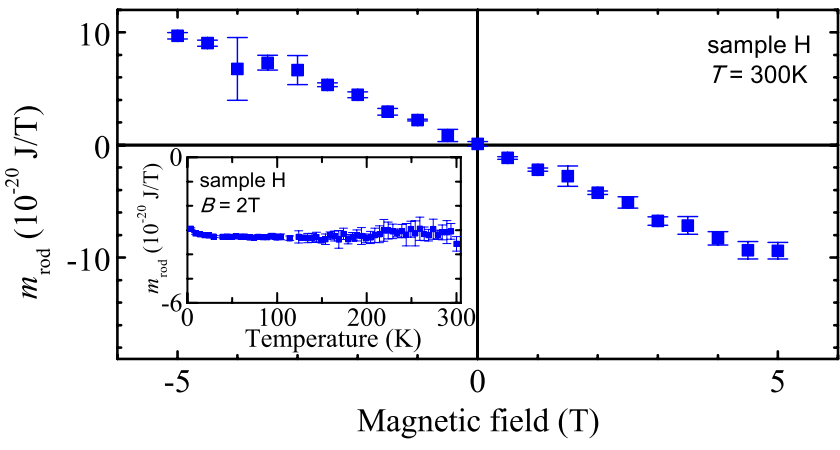

FIG. 4 (color online). Magnetic moment per rod versus magnetic field at $300 \mathrm{~K}$; a linear slope is visible. The data points and error bars are experimentally background-corrected values [15]. Similarly, the inset shows the magnetic moment $m_{\text {rod }}$ at $2 \mathrm{~T}$ versus temperature. The field dependence was measured four times, which resulted in an average susceptibility of $\chi_{V}=$ $(-4.9 \pm 1.2) \times 10^{-4}$.
SQUID results, therefore, prove that the degree of alignment is predominantly due to the Au cores.

To understand the enhanced magnetism of the $\mathrm{Au}$ nanorods, we first discuss sample $H$, of which we have a full set of experimental data. It exhibits an anomalous temperature-independent, diamagnetic response (Fig. 4), which is compatible with the magnetic alignment signal that does not show any hysteresis and saturation, even at $32 \mathrm{~T}$ (Fig. 3). The dimensions of these nanorods are much larger than the particles for which para- or ferromagnetism have been observed (typically smaller than $5 \mathrm{~nm}$ ). Furthermore, most ferro- and paramagnetic NPs are capped by strongly interacting molecules, such as thiols, binding with strong Au-S bonds, leading to a reduction of the free electron density and eventually to the disappearance of the SPR [6]. In our case, both CTAB and (thiolated) mPEG-SH cappings left the electronic surface properties unchanged (reduction in free electron density $<1 \%$ [15]) and resulted in a similar magnetic response. This rules out most of the previous suggested mechanisms, involving Au-ligand interactions, to explain the enhanced diamagnetism observed here. Therefore, we believe the enhanced diamagnetism to be of orbital nature [2,11].

The dimensions of the sample $H$ nanorods ( $W=31 \mathrm{~nm}$, $L=73 \mathrm{~nm}$ ) are comparable to the room temperature elastic mean free path $l_{e}(\sim 60 \mathrm{~nm}[23,24])$ and thermal length $L_{T}=\hbar v_{f} / \pi k_{B} T(\sim 10 \mathrm{~nm})$ of bulk Au ( $\hbar$ is Planck's constant, $v_{f}$ is the electron Fermi velocity) and much smaller than the low temperature $(5 \mathrm{~K}) l_{e}$ and $L_{T}$. The temperature-independent $\chi_{V}$ measured by SQUID thus strongly suggest that even sample $H$ rods (our largest) are in the mesoscopic $\left(R<L_{T}\right)$ and ballistic $\left(R<l_{e}\right)$ regimes up to room temperature, where $R=V^{1 / 3}$ is the characteristic size of the rods. Mesoscopic fluctuations of the orbital magnetic susceptibility of finite size metallic systems can be very large and have been the subject of quite intensive theoretical investigations [14,30]. For an individual metallic nanostructure, the magnetic susceptibility can oscillate between negative and positive values, as a function of $k_{F} R$ ( $k_{F}$ is the Fermi wave vector), exhibiting very large values of up to 100 times the Landau susceptibility of a bulk free electron gas. For an ensemble consisting of nonidentical nanosystems, as we consider here, where the variations in $k_{F} R$ are large $(>10 \%)$, these oscillations disappear, but the susceptibility does not average out to zero. The remaining ensemble susceptibility $\chi_{V}$ can still be substantial and is typically paramagnetic, both in the diffusive [30] and the ballistic [14] regimes. Experimental evidence for this enhanced paramagnetic susceptibility has been found for micrometer sized GaAs squares [34]. It is, therefore, likely that orbital magnetism in high quality single-crystalline metallic nanoparticles can be quite significant, although a full calculation is not available in literature. Such a calculation should include several new ingredients to explain our results. First of all, it should take into account spin-orbit coupling, which is known to be strong in Au $[35,36]$ and 
which may result in a change of sign of the orbital magnetism (from para- to diamagnetic) analogous to the change of sign of the magnetoconductance under the influence of spin-orbit coupling [37]. Second, the possible effects of surface states should be taken into account [27,28], especially when considering smaller NPs. Third, the actual geometry of the nanoparticles plays an important role in their final orbital magnetism because of the contributions of the different electron trajectories, which can be either regular or chaotic [14,29,30,38]. Most probably, the latter two points are crucial in order to understand the increasing value of $\Delta \chi_{V}$ with decreasing volume and increasing aspect ratio, because for those samples the surface/volume ratio increases [dashed black line in Fig. 3(b)].

In conclusion, we have observed a giant magnetic susceptibility of Au nanorods in solution using an optically detected magnetic alignment technique. We speculate that this enhanced magnetism results from orbital magnetism of the free electrons in the Au core. We anticipate that singlecrystalline metal NPs provide a versatile experimental system to better understand mesoscopic fluctuations in the orbital magnetic susceptibility of confined electron systems in different quantum transport regimes.

We thank P. W. Albers, L. P. Nelemans, and J. M. H. van Velsen for technical assistance, and Z. Gu for his contribution at the early stages of the project. Part of this work has been supported by the Foundation for Fundamental Research on Matter (FOM) with financial support from the Netherlands Organisation for Scientific Research (NWO), EuroMagNETII under EU Contract No. 228043, and the ERC Project SiMoSoMa.

*P.Christianen@science.ru.nl

[1] E. Guerrero, M. A. Muñoz Márquez, M. A. García, P. Crespo, E. Fernández-Pinel, A. Hernando, and A. Fernández, Nanotechnology 19, 175701 (2008).

[2] R. Gréget, G. L. Nealon, B. Vileno, P. Turek, C. Mény, F. Ott, A. Derory, E. Voirin, E. Rivière, A. Rogalev et al., ChemPhysChem 13, 3092 (2012).

[3] Y. Yamamoto, T. Miura, M. Suzuki, N. Kawamura, H. Miyagawa, T. Nakamura, K. Kobayashi, T. Teranishi, and H. Hori, Phys. Rev. Lett. 93, 116801 (2004).

[4] Y. Negishi, H. Tsunoyama, M. Suzuki, N. Kawamura, M. M. Matsushita, K. Maruyama, T. Sugawara, T. Yokoyama, and T. Tsukuda, J. Am. Chem. Soc. 128, 12034 (2006).

[5] J. Bartolomé, F. Bartolomé, L. M. García, A. I. Figueroa, A. Repollés, M. J. Martínez, F. Luis, C. Magén, S. Selenska-Pobell, F. Pobell et al., Phys. Rev. Lett. 109, 247203 (2012).

[6] P. Crespo, R. Litrán, T. C. Rojas, M. Multigner, J. M. de la Fuente, J. C. Sánchez-López, M. A. García, A. Hernando, S. Penadés, and A. Fernández, Phys. Rev. Lett. 93, 087204 (2004).

[7] J. S. Garitaonandia, M. Insausti, E. Goikolea, M. Suzuki, J. D. Cashion, N. Kawamura, H. Ohsawa, I. Gil de Muro, K. Suzuki, F. Plazaola et al., Nano Lett. 8, 661 (2008).
[8] M. Suda, N. Kameyama, M. Suzuki, N. Kawamura, and Y. Einaga, Angew. Chem., Int. Ed. Engl. 47, 160 (2008).

[9] A. Hernando, P. Crespo, and M. A. García, Phys. Rev. Lett. 96, 057206 (2006).

[10] S. Trudel, Gold Bull. (London) 44, 3 (2011).

[11] G. L. Nealon, B. Donnio, R. Greget, J.-P. Kappler, E. Terazzi, and J.-L. Gallani, Nanoscale 4, 5244 (2012).

[12] U. Kreibig and M. Vollmer, Optical Properties of Metal Clusters (Springer, Berlin, 1995).

[13] C. Bohren and D. Huffman, Absorption and Scattering of Light by Small Particles (Wiley, New York, 1998).

[14] K. Richter, D. Ullmo, and R. A. Jalabert, Phys. Rep. 276, 1 (1996).

[15] See Supplemental Material at http://link.aps.org/ supplemental/10.1103/PhysRevLett.111.127202 for additional high-resolution TEM images, full derivations, additional data, and analysis.

[16] C. Sönnichsen and A. P. Alivisatos, Nano Lett. 5, 301 (2005).

[17] Q. Liu, Y. Cui, D. Gardner, X. Li, S. He, and I. I. Smalyukh, Nano Lett. 10, 1347 (2010).

[18] P. V. Ruijgrok, N.R. Verhart, P. Zijlstra, A.L. Tchebotareva, and M. Orrit, Phys. Rev. Lett. 107, 037401 (2011).

[19] P. Zijlstra, M. van Stee, N. Verhart, Z. Gu, and M. Orrit, Phys. Chem. Chem. Phys. 14, 4584 (2012).

[20] P. Zijlstra, C. Bullen, J. Chon, and M. Gu, J. Phys. Chem. B 110, 19315 (2006).

[21] B. Nikoobakht and M. El-Sayed, Chem. Mater. 15, 1957 (2003).

[22] P. Zijlstra and M. Orrit, Rep. Prog. Phys. 74, 106401 (2011).

[23] CRC Handbook of Chemistry and Physics (Taylor and Francis Group, London, 2013), 94th ed.

[24] S. Vonsovsky and M. Katsnelson, Quantum Solid State Physics (Springer Series in Solid-State Sciences, Berlin, 1989).

[25] I. O. Shklyarevskiy, P.C.M. Christianen, E. Aret, H. Meekes, E. Vlieg, G. Deroover, P. Callant, L. van Meervelt, and J. C. Maan, J. Phys. Chem. B 108, 16386 (2004).

[26] D. Craig, Magnetism. Principles and Applications (Wiley, New York, 1995).

[27] P. Avouris and I.-W. Lyo, Science 264, 942 (1994).

[28] F. Reinert, G. Nicolay, S. Schmidt, D. Ehm, and S. Hüfner, Phys. Rev. B 63, 115415 (2001).

[29] J. Ma and K. Nakamura, Phys. Rev. B 60, 10676 (1999).

[30] B. L. Altshuler, Y. Gefen, Y. Imry, and G. Montambaux, Phys. Rev. B 47, 10335 (1993).

[31] H. Yonemura, S. Natsuko, J. Suyama, and S. Yamada, J. Photochem. Photobiol., A 220, 179 (2011).

[32] J. S. Clawson, G. P. Holland, and T. M. Alam, Phys. Chem. Chem. Phys. 8, 2635 (2006).

[33] T. M. Alam and S. K. McIntyre, Langmuir 24, 13890 (2008).

[34] L. P. Levy, G. Dolan, J. Dunsmuir, and H. Bouchiat, Phys. Rev. Lett. 64, 2074 (1990).

[35] S. LaShell, B. A. McDougall, and E. Jensen, Phys. Rev. Lett. 77, 3419 (1996).

[36] M. Hoesch, M. Muntwiler, V. N. Petrov, M. Hengsberger, L. Patthey, M. Shi, M. Falub, T. Greber, and J. Osterwalder, Phys. Rev. B 69, 241401 (2004).

[37] C. W. J. Beenakker, Rev. Mod. Phys. 69, 731 (1997).

[38] W. P. Halperin, Rev. Mod. Phys. 58, 533 (1986). 